\title{
Aggressive Osteoblastoma of the Maxilla: A Case Report and Review of the Literature
}

\author{
Christine Harrington $\cdot$ Brent T. Accurso • \\ John R. Kalmar • Obiajulu H. Iwenofu • \\ Amit Agrawal · Carl M. Allen • Marino E. Leon
}

Received: 10 November 2010/Accepted: 8 December 2010/Published online: 23 December 2010

(C) Springer Science+Business Media, LLC 2010

\begin{abstract}
Aggressive osteoblastoma is a rare primary bone neoplasm with the potential for local invasion and recurrence. While the vertebrae or long bones are most commonly affected, few well-documented cases have been reported in the jaws. A 25-year-old man presented with a palatal mass of several months' duration. He reported the lesion had undergone gradual enlargement and, while generally asymptomatic, had recently become increasingly painful. An incisional biopsy was interpreted as "osteoblastic neoplasm" most suggestive of osteoblastoma. However, final diagnosis was deferred until the resection specimen could be evaluated. Following partial maxillectomy, histopathologic examination revealed a proliferation of large epithelioid cells with eccentric nuclei and prominent nucleoli associated with broad, irregular deposits of
\end{abstract}

C. Harrington $(\varangle) \cdot$ B. T. Accurso · C. M. Allen

Oral and Maxillofacial Pathology, The Ohio State University

College of Dentistry, 305 W. 12th Avenue, Columbus,

OH 43210, USA

e-mail: harrington.162@osu.edu

\section{J. R. Kalmar}

Division of Oral and Maxillofacial Surgery, Pathology and Anesthesiology, The Ohio State University College of Dentistry, 305 W. 12th Avenue, Columbus, OH 43210, USA

\section{O. H. Iwenofu}

Divisions of Head \& Neck Pathology and Soft Tissue \& Bone Pathology, Department of Pathology, The Ohio State University, Columbus, OH, USA

\section{A. Agrawal}

Department of Otolaryngology, Head and Neck Surgery, The Ohio State University, Columbus, OH, USA

C. M. Allen

Department of Pathology, College of Medicine and Public Health, The Ohio State University, Columbus, OH, USA osteoid and trabeculae of bone. The lesional cells exhibited minimal pleomorphism with infrequent, normal-appearing mitotic figures and numerous osteoclast-like giant cells were observed within an associated loose fibrovascular stroma. Transformation of "blue bone" to more organized eosinophilic trabeculae of woven bone was noted at the periphery of the lesion and there was no evidence of invasion. A diagnosis of aggressive osteoblastoma was made. Previous reports of gnathic aggressive osteoblastoma are reviewed and the features that distinguish this process from conventional osteoblastoma or osteoblastoma-like osteosarcoma are presented.

Keywords Aggressive osteoblastoma - Gnathic . Maxilla $\cdot$ Osteoblastoma-like osteosarcoma

\section{E. Leon}

Anatomic Pathology, Moffitt Cancer Center, Tampa, FL, USA

M. E. Leon

Department of Oncologic Sciences, University of South Florida College of Medicine, Tampa, FL, USA

M. E. Leon

Department of Pathology and Cell Biology, University of South Florida College of Medicine, Tampa, FL, USA

M. E. Leon

Division of Head \& Neck Pathology, Department of Pathology, The Ohio State University, Columbus, OH, USA 


\section{Introduction}

Osteoblastoma was first described in the English literature by Jaffe and Mayer in 1932. They reported an osteoblast-rich osteoid tissue-forming tumor of the metacarpal bone and suggested that Virchow may have reported the same lesion in 1863 [1]. In 1956, Jaffe and Lichtenstein independently proposed the term "benign osteoblastoma" to identify an osteoblastic osteoid-forming lesion similar to osteoid osteoma, but having a greater growth potential [2,3]. Since that time, lesions histopathologically similar to benign or conventional osteoblastoma $(\mathrm{CO})$ have been reported that have demonstrated a locally destructive growth pattern and a high recurrence rate after curettage. In 1972 Dorfman included osteoblastoma in a review of malignant transformation of benign bone lesions. In addition to discussing malignant change of osteoblastoma, he examined 23 cases of osteoblastoma from his lab. He noted four of these cases exhibited recurrent behavior and histologic features that would merit the name "aggressive osteoblastoma," although the characteristic histologic features were not specified [4]. Dorfman and Weiss described aggressive osteoblastoma (AO) as an entity of borderline osteoblastic tumors in 1984 and suggested that the presence of epithelioid osteoblasts was a distinctive histologic feature [5]. In his textbook of skeletal tumors, Dorfman subsequently defined AO as a "...rare tumor that represents a borderline lesion between benign osteoblastoma and osteosarcoma. These tumors are likely to recur, do not metastasize, and are characterized microscopically by the presence of so-called epithelioid osteoblasts" [6].

Conventional osteoblastoma (CO) accounts for $<1 \%$ of all primary bone tumors. It most commonly affects males during the second decade and often arises within the vertebral column and long bones [7, 8]. Approximately $10-12 \%$ of $\mathrm{CO}$ occur in the maxillofacial skeleton, especially the mandible [7-9]. Due to its relative rarity, the incidence and distribution of aggressive osteoblastoma (AO) are currently unknown [11]. Reports of gnathic AO are exceptionally rare $[10,12,13]$. Both $\mathrm{CO}$ and $\mathrm{AO}$ often present with local pain and swelling; however, $\mathrm{AO}$ affects an older age group than $\mathrm{CO}$, usually arising in the third or fourth decade $[6,7]$. The radiographic appearance of AO is similar to $\mathrm{CO}$, consisting of a circumscribed lytic defect sometimes surrounded by a sclerotic rim, although a more aggressive appearance including significant cortical expansion and destruction can be seen. Intralesional radiopacities of varying amount and density have been described [6, 7, 12]. Importantly, AO lesions tend to be clinically and radiographically larger $(\geq 4 \mathrm{~cm})$ than lesions of $\mathrm{CO}(<4 \mathrm{~cm})$ [6]. In a review of 306 cases of osteoblastoma, 24\% had large epithelioid osteoblasts. These osteoblasts were the predominant cellular element (at least
$75 \%)$, in approximately $10 \%(n=30)$ of the cases. The authors examined these tumors as a separate group in order to evaluate the purported aggressiveness of such osteoblastomas. Although $40 \%$ of these cases had prominent mitotic activity and two-thirds had a multifocal growth pattern, the authors concluded that the presence of epithelioid osteoblasts alone was not a reliable predictor of aggressive behavior. Upon follow up, only 4 of the 30 cases exhibited clinically aggressive behavior; three had recurrences and one had two additional recurrences [7]. A subsequent report of 55 additional cases also concluded that clinically aggressive behavior in osteoblastoma did not seem related solely to the presence of epithelioid osteoblasts [8].

Given these findings, several authors have suggested that clinical aggressiveness in osteoblastoma appears more dependent on the precise location and size of the tumor than on its microscopic features [7,8]. Therefore, while epithelioid osteoblasts are critical to the diagnosis of $\mathrm{AO}$, tumor size and location seem to be more important considerations with regard to treatment and prognosis. Tumors greater than $4 \mathrm{~cm}$ in diameter or those located in anatomic sites that impact the surgeon's ability to completely remove lesional tissue are more likely to recur or cause local tissue destruction $[7,8,12]$. Proper management of patients with AO requires careful correlation of clinical, radiographic and three-dimensional imaging studies.

\section{Report of Case}

A 25-year-old man presented to the emergency room of The Ohio State University Medical Center with a gradually enlarging palatal mass of several months' duration. The mass had been generally asymptomatic, but recently had grown even larger and become increasingly painful. Clinically the lesion appeared as an ulcerated, firm, exophytic mass palatal to teeth \#12-\#16, approximately $4 \mathrm{~cm}$ in diameter. A fetid odor was noticed, and left infraorbital swelling was present. The maxillary left posterior teeth were mobile and sensitive to percussion. The maxillary left buccal gingiva was swollen and a purulent exudate was observed. Panoramic radiographic examination showed an ill-defined radiolucency with internal radiodensities extending from approximately the left maxillary lateral incisor to the left maxillary tuberosity. The axial CT showed a large expansile calcified mass involving the left side of the hard palate and left maxillary alveolar ridge, eroding through the medial and inferior walls of the left maxillary sinus (Fig. 1). The lesion involved several left posterior maxillary teeth from the first premolar to the first molar area. In a comment given as a part of the CT scan report, the radiologist expressed a concern for 


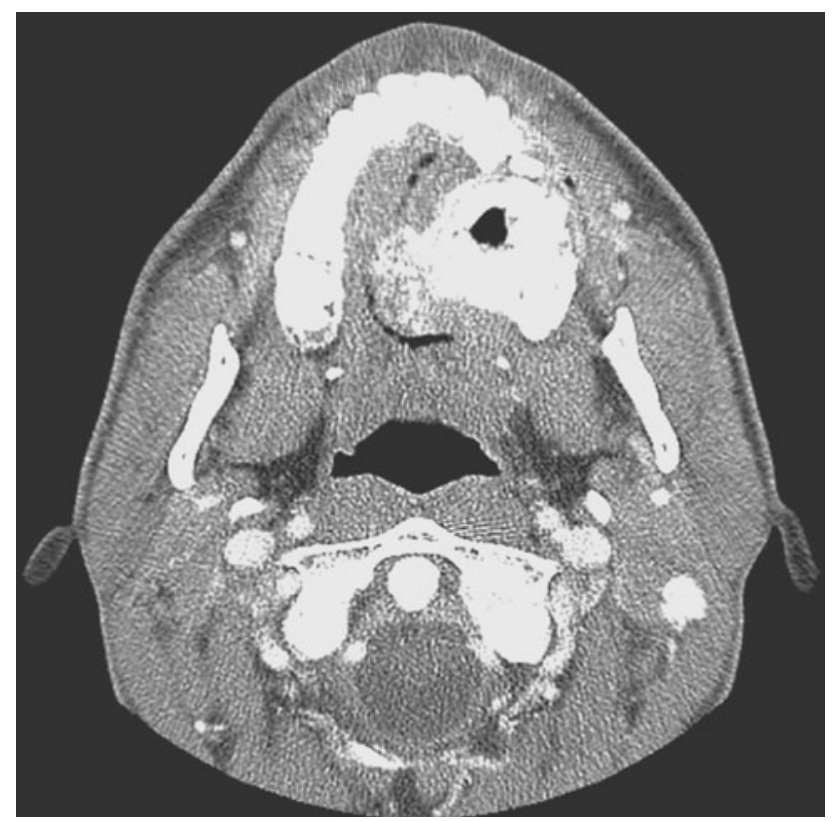

Fig. 1 Axial CT

osteosarcoma. Approximately 2 weeks later, an incisional biopsy of the palatal mass was performed. At that time, a diagnosis of "osteoblastic neoplasm" was rendered, and although the pathologist favored a diagnosis of osteoblastoma, osteoblastoma-like osteosarcoma could not be excluded. The definitive diagnosis was deferred until examination of the resected specimen. One month later the patient underwent a partial maxillectomy. The final diagnosis was aggressive osteoblastoma. Eight months postoperatively, there has been no evidence of recurrence and the patient has been fitted successfully with a palatal obturator.

\section{Results}

Histopathologic examination of the resected specimen revealed a proliferation of large epithelioid cells with eccentric nuclei and prominent nucleoli associated with broad, irregular deposits of osteoid and trabeculae of bone (Fig. 2). Sheets of these epithelioid osteoblasts filled the intertrabecular spaces and rimmed the trabeculae of bone (Fig. 3). Mitotic figures were uncommon and no atypical mitoses were seen. Osteoclast-like giant cells were readily identifiable within an associated loose fibrovascular stroma. Peripheral maturation, represented by transformation from "blue bone" to a more organized pattern of eosinophilic trabeculae of woven bone, was noted at the tumor borders (Fig. 4). No entrapment or permeation of host lamellar bone was identified. The surgical margins

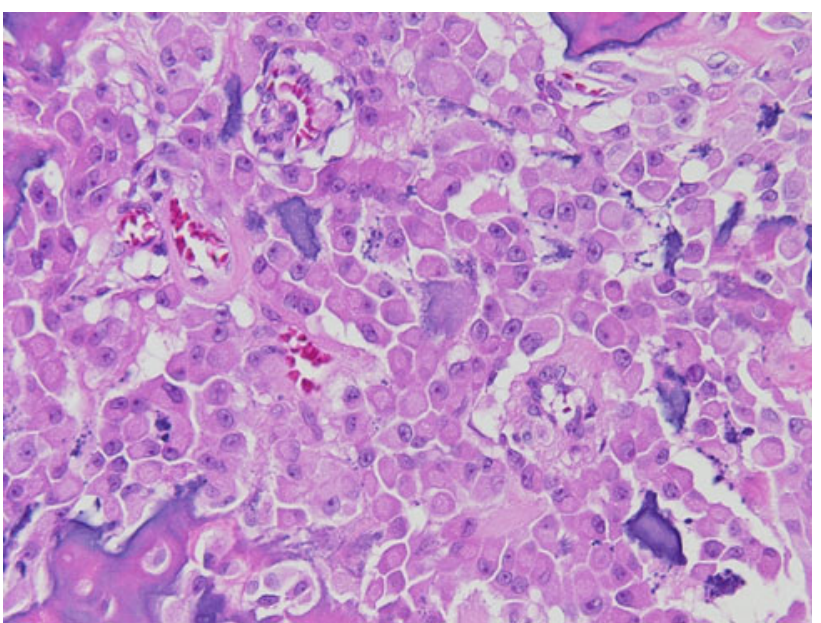

Fig. 2 Sheet of epithelioid osteoblasts with cytoplasmic clearing and eccentric nuclei

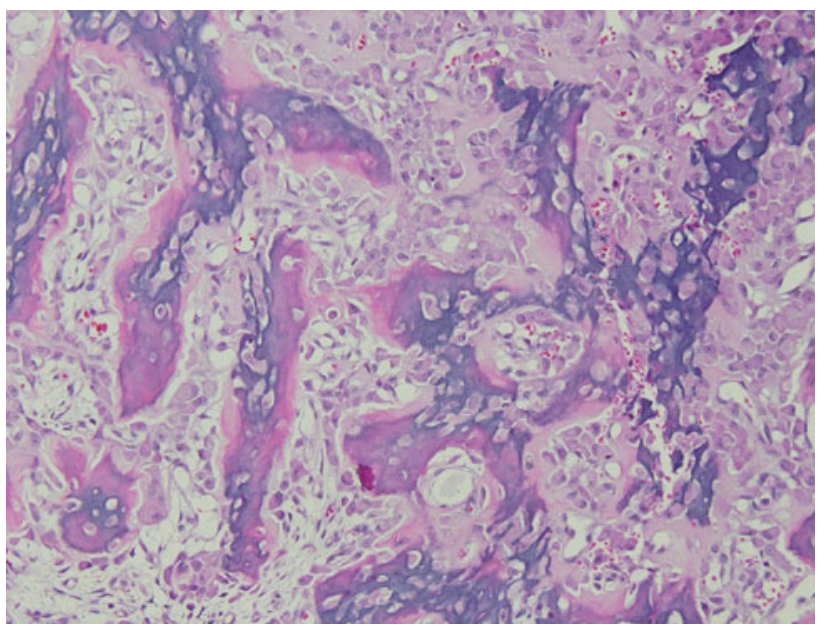

Fig. 3 Immature trabeculae with osteoblastic rimming and prominent osteocytes

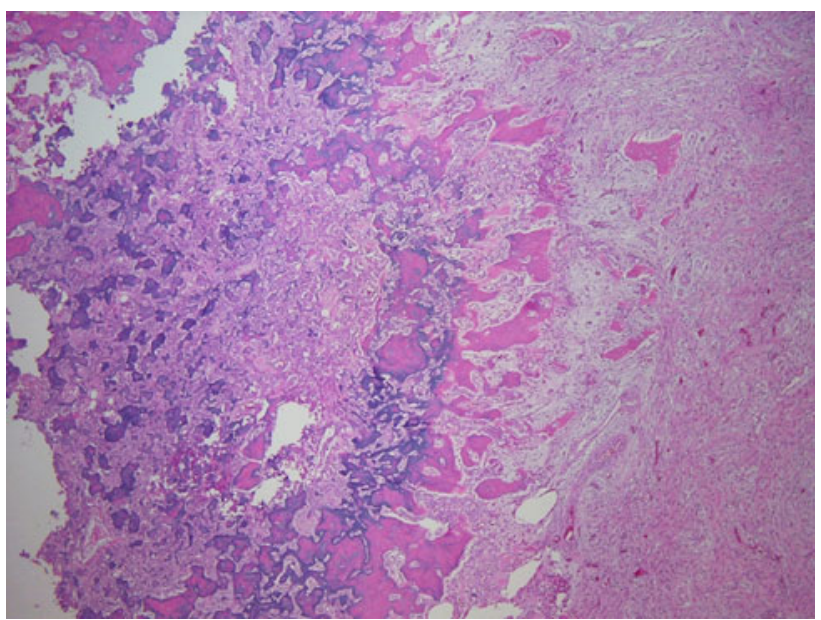

Fig. 4 Zonation of bone formation with peripheral maturation 
were clear. Based on these features, a diagnosis of aggressive osteoblastoma was made.

\section{Discussion}

The microscopic differential diagnosis for $\mathrm{AO}$ includes $\mathrm{CO}$ and osteoblastoma-like osteosarcoma (OB-like OS) (Table 1). Subtle differences and overlapping features among these lesions can be challenging for the pathologist and a small biopsy specimen may preclude definitive diagnosis, as was encountered in our case [7, 14, 15]. The hallmark of $\mathrm{AO}$ is a prominent population of plump epithelioid osteoblasts. These cells are slightly larger than the osteoblasts of $\mathrm{CO}$ and not only rim the lesional trabeculae but may form solid intertrabecular sheets. In addition, the trabeculae of AO are often wider and more irregular than those of $\mathrm{CO}$ with osteoid deposition that can vary from solid to lacelike $[5,6]$. The prominent cytoplasm of the epithelioid osteoblasts typically appears eosinophilic and may exhibit a central area of clearing $[5,6]$. The eccentric nucleus is round to oval and often contains a single nucleolus. Mitoses are usually rare to absent, and when noted are normal in appearance [6]. Osteoclast-like giant cells are frequently noted. The associated stroma can vary from loosely fibrovascular to more cellular; however, the vascularity in lesions of AO tends to be less notable than in CO $[5,7]$. Secondary aneurysmal bone cyst formation may be noted [6-8]. Microscopic features once thought to be worrisome for malignancy or aggressive behavior, including: focal presence of chondroid matrix, spiculated blue bone, fine lace-like osteoid, bizarre cells and prominent mitotic activity; have not been found to significantly impact either behavior or outcome [7, 8, 15, 16].

While the borders of both $\mathrm{CO}$ and $\mathrm{AO}$ can be welldefined, the host-tumor interface of $\mathrm{CO}$ is typically better demarcated than in $\mathrm{AO}$, with distinct peripheral maturation, or zonation, from "blue bone" to organized trabeculae of eosinophilic woven bone [7, 14, 15]. Importantly, permeation into or entrapment of host bone is not seen with either $\mathrm{CO}$ or $\mathrm{AO}$, a critical distinction with regard to benign versus malignant clinical behavior [7, 12, 15-17].

Osteoblastoma-like osteosarcoma (OB-like OS) is the major concern in the differential diagnosis of aggressive osteoblastoma. As with $\mathrm{AO}$, pain is a frequent presenting symptom [5, 7, 14-16, 18]. OB-like OS was first reported by Bertoni who evaluated a series of seventeen patients ranging in age from 11 to 58 years old. Seven of the seventeen patients died of their disease [14]. In a later series, Bertoni et al. reported an additional 11 cases (patient ages from 11 to 47 years) OB-like OS cases [15]. OB-like OS is a low-grade osteosarcoma with radiographic features that can vary from lytic to sclerotic to mixed. Lesion borders can be well defined, suggesting a benign process, or can be ill defined and have cortical destruction [14, 15]. As with AO, OB-like OS can have large epithelioid osteoblasts, but may also show spindled cells with hyperchromatic nuclei, prominent nucleoli and deeply-stained cytoplasm. Occasional atypical mitoses are found. A more irregular, occasionally lace-like pattern of the lesional trabeculae is seen, and anaplastic cells can be found trapped within the osteoid. The stroma is solid and cellular. Most importantly, the

Table 1 Comparison of conventional osteoblastoma (CO), aggressive osteoblastoma (AO) and osteoblastoma-like osteosarcoma (OB-like OS)

\begin{tabular}{|c|c|c|c|c|c|}
\hline & $\begin{array}{l}\text { Cytologic features: } \\
\text { osteoblasts }\end{array}$ & $\begin{array}{l}\text { Nuclear features: } \\
\text { osteoblasts }\end{array}$ & $\begin{array}{l}\text { Features of osteoid/ } \\
\text { woven bone }\end{array}$ & $\begin{array}{l}\text { Intertrabecular } \\
\text { features }\end{array}$ & Tumor borders \\
\hline $\begin{array}{l}\text { Osteoblastoma } \\
\text { (Conventional) }\end{array}$ & $\begin{array}{l}\text { Polygonal } \\
\text { Occasionally "epithelioid" } \\
\text { Moderate amount of } \\
\text { cytoplasm }\end{array}$ & $\begin{array}{l}\text { Round to oval } \\
\text { Prominent } \\
\text { nucleoli } \\
\text { Rare to absent } \\
\text { mitoses }\end{array}$ & $\begin{array}{l}\text { Long anastomosing } \\
\text { trabeculae } \\
\text { Osteoblastic } \\
\text { rimming }\end{array}$ & $\begin{array}{l}\text { Prominent } \\
\text { vascularity }\end{array}$ & Well- demarcated \\
\hline $\begin{array}{l}\text { Aggressive } \\
\text { osteoblastoma }\end{array}$ & $\begin{array}{l}\text { Epithelioid } \\
\text { Larger and more numerous } \\
\text { Eosinophilic cytoplasm, } \\
\text { often with a clear area }\end{array}$ & $\begin{array}{l}\text { Eccentric } \\
\text { Round to oval } \\
\text { Prominent } \\
\text { nucleoli } \\
\text { Occasional } \\
\text { typical mitoses }\end{array}$ & $\begin{array}{l}\text { Larger more } \\
\text { irregular } \\
\text { trabeculae } \\
\text { Osteoblastic } \\
\text { rimming }\end{array}$ & $\begin{array}{l}\text { Less vascular } \\
\text { Sheets of } \\
\text { "epithelioid" } \\
\text { osteoblasts }\end{array}$ & $\begin{array}{l}\text { Peripheral maturation } \\
\text { without sharp demarcation }\end{array}$ \\
\hline $\begin{array}{l}\text { Osteoblastoma-like } \\
\text { osteosarcoma }\end{array}$ & $\begin{array}{l}\text { Large round to oval, or } \\
\text { spindled } \\
\text { May be epithelioid } \\
\text { Deeply staining cytoplasm }\end{array}$ & $\begin{array}{l}\text { Hyperchromatic } \\
\text { Prominent } \\
\text { nucleoli } \\
\text { Occasional } \\
\text { atypical } \\
\text { mitoses }\end{array}$ & $\begin{array}{l}\text { Irregular pattern of } \\
\text { trabeculae } \\
\text { Anaplastic cells } \\
\text { within osteoid }\end{array}$ & $\begin{array}{l}\text { Solid cellular } \\
\text { stroma }\end{array}$ & Infiltration of host bone \\
\hline
\end{tabular}


border of the tumor shows infiltration and entrapment of host bone [6, 7, 14-16, 18]. Biopsy should include the peripheral area of cortical bone surrounding the tumor, particularly when radiographic findings suggest an aggressive lesion [18].

Our review of the literature found four well-documented gnathic aggressive osteoblastomas. In 1989, Ohkubo et al. reported a case in the maxilla of a 6-year-old boy. The tumor surrounded the adjacent deciduous second molar and expanded the left antral floor. It was treated by en bloc resection, and there was no evidence of recurrence or metastasis four years post-operatively. The authors concluded that the division of osteoblastoma into benign and aggressive subgroups may be artificial and unnecessary, suggesting the high recurrence rate may be due to incomplete or piecemeal excision of the lesion [13]. Two cases of $\mathrm{AO}$ of the mandible simulating calcifying epithelial odontogenic tumor were reported by Vigneswaran et al. in 2001. One patient was a 21-year-old male, the other a 12 -year-old female. Osteoid in these tumors was eosinophilic, amorphous and homogenous, resembling amyloid, but did not stain with Congo red. Areas of the extracellular matrix showed varying degrees of calcification. Neither case showed easily recognizable trabecular neoplastic bone formation or chondroid differentiation. An osteoblastic origin of the epithelioid cells was confirmed immunohistochemically by their immunoreactivity with antibodies directed against osteocalcin. The authors cautioned that being aware of these previously underemphasized features must be considered to reach an accurate diagnosis [12]. Lypka et al. in 2008 reported AO of the mandible in a 33-year-old male and emphasized the importance of correlation among the gross, radiographic and microscopic features to arrive at the correct diagnosis and to develop the proper surgical plan [10]. Microscopically, all of these cases exhibited epithelioid osteoblasts, zonation and no atypical mitoses. All of them had localized aggressive behavior, but none had evidence of metastasis. Treatment for all of the patients consisted of surgery with no adjuvant radiation or chemotherapy (Table 2).

Few genetic studies have been published regarding markers to aid in the diagnosis of osteoblastoma and to help differentiate AO from OB-like OS. A recent publication by Giannico et al. reported a novel three-way translocation involving chromosomes 1, 2 and 14 in an osteoblastoma of a 23-year-old female [17]. Other cytogenetic analyses have been done on $\mathrm{CO}$ resulting in a range of findings. Baker et al. reported a case of a unique chromosomal aberration in aggressive osteoblastoma of the femoral diaphysis of a 12-year-old girl. The cytogenetic analysis performed in that case showed that of the 22 metaphases analyzed, 20 revealed a pseudodiploid clone with a balanced translocation between chromosomes 4, 7, and 14 [19]. Although the balanced translocation found was unique, the authors could not unequivocally state whether the abnormality provided any insight into differentiating $\mathrm{CO}, \mathrm{AO}$, or OB-like OS. They concluded that cytogenetic studies are not yet useful for either diagnostic or prognostic purposes with these challenging lesions and concurred with others that careful correlation of clinical, radiographic and histologic findings is paramount to proper patient management [19].

In conclusion, we have presented a case of aggressive osteoblastoma of the maxilla. In the jaws, AO can present with pain, swelling, and loosening of teeth, mimicking a dental infection or osteomyelitis [9, 12]. As the tumor grows, the radiographic appearance, in addition to the pain, can be suggestive of osteosarcoma [12]. Because AO has a spectrum of appearances microscopically, the diagnosis can be a challenge. Appropriate treatment for AO is surgical resection with reconstruction and prosthetic replacement as needed [10].

Historically the nomenclature of osteoid-forming lesions has been controversial due to their overlapping features and because the relative rarity of the lesions has precluded numerous large case series to better define their biologic

Table 2 Comparison of current case with previously reported cases of gnathic aggressive osteoblastoma

\begin{tabular}{|c|c|c|c|c|c|}
\hline & \multirow[t]{2}{*}{ Current case (2009) } & \multirow[t]{2}{*}{ Ohkubo et al. (1989) } & \multicolumn{2}{|c|}{ Vigneswaran et al. (2001) } & \multirow[t]{2}{*}{ Lypka et al. (2008) } \\
\hline & & & Case \#1 & Case \#2 & \\
\hline Age & 25 years & 6 years & 21 years & 12 years & 33 years \\
\hline Gender & Male & Male & Male & Female & Male \\
\hline Site & Posterior maxilla & Posterior maxilla & Posterior mandible & Posterior mandible & Body of mandible \\
\hline Size & $3.2 \times 4.1 \times 2.9 \mathrm{~cm}^{*}$ & $3.0 \times 2.0 \times 2.0 \mathrm{~cm}$ & $7.5 \times 6.2 \times 4.2 \mathrm{~cm}$ & $4.5 \times 3.0 \times 0.7 \mathrm{~cm}$ & $* *$ \\
\hline Symptoms & Swelling, pain & Swelling, pain & Swelling, pain & Swelling & Swelling, pain \\
\hline Treatment & En bloc resection & En bloc resection & En bloc resection & En bloc resection & Segmental resection \\
\hline Follow-up & 8 mos. - no recurrence & 4 years.-no recurrence & 2 years.-no recurrence & 22 mos.-no recurrence & 1 year.-no recurrence \\
\hline
\end{tabular}

* Size measured on CT with comment of inability to rule out further extension superiorly in the maxillary sinus

** Size not reported 
potential. Most agree that osteoid osteoma (OO) and $\mathrm{CO}$ are separate but related entities. Their separation is primarily based on size and location, together with degree of reactive sclerosis of the surrounding bone and associated pain [5]. Similar features appear to most accurately distinguish CO from AO. Should "aggressive osteoblastoma" continue to be applied as a histopathologic diagnosis when the lesions to which it applies do not consistently exhibit a more aggressive biologic behavior? Or should the diagnosis of $\mathrm{AO}$ be reserved for osteoblastic, osteoid-forming tumors larger than $4 \mathrm{~cm}$ in greatest diameter? Ultimately, the pathologist is responsible for a diagnosis that is not only accurate, but also communicates information which will be helpful in patient management. As with the separation of osteoid osteoma from conventional osteoblastoma, the distinction of AO from $\mathrm{CO}$ may be currently best achieved through clinical and pathologic correlation.

\section{References}

1. Jaffe H, Mayer L. An osteoblastic osteoid tissue-forming tumor of a metacarpal bone. Arch Surg. 1932;24:550-64.

2. Jaffe H. Benign osteoblastoma. Bull Hosp Jt Dis. 1956;17: 141-51.

3. Lichtenstein L. Benign osteoblastoma: a category of osteoid-and bone-forming tumors other than classical osteoid osteoma, which may be mistaken for giant-cell tumor or osteogenic sarcoma. Cancer. 1956;9:1044-52.

4. Dorfman H. Malignant transformation of benign bone lesions. Proc Natl Cancer Conf. 1972;7:901-13.

5. Dorfman H, Weiss S. Borderline osteoblastic tumors: problems in the differential diagnosis of aggressive osteoblastoma and lowgrade osteosarcoma. Semin Diagn Pathol. 1984;1:215-34.
6. Dorfman HD, Czerniak B. Bone Tumors. St. Louis: Mosby, Inc. 1998. p 103-21.

7. Lucas D, Unni KK, McLeod R, et al. Osteoblastoma: a clinicopathologic study of 306 cases. Hum Pathol. 1994;25:117-34.

8. Rocca CD, Huvos AG. Osteoblastoma: varied histological presentations with a benign clinical course: an analysis of 55 cases. Am J Surg Pathol. 1996;20:841-50.

9. Rawal Y, Angiero F, Allen CM, et al. Gnathic osteoblastoma: clinicopathologic review of seven cases with long-term followup. Oral Oncol. 2006;42:123-30.

10. Lypka MA, Goos RR, Yamashita D-DR, Melrose R. Aggressive osteoblastoma of the mandible. Int J Oral Maxillofac Surg. 2008; 37:675-8.

11. Nishida K, Doita M, Kawahara N, et al. Total en bloc spondylectomy in the treatment of aggressive osteoblastoma of the thoracic spine. Orthopedics. 2008;31(4):403-6.

12. Vigneswaran N, Fernandes R, Rodu B, et al. Aggressive osteoblastoma of the mandible closely simulating calcifying epithelial odontogenic tumor. Pathol Res Pract. 2001;197:569-76.

13. Ohkubo T, Hernandez JC, Ooya K. Krutchkoff. Aggressive osteoblastoma of the maxilla. Oral Surg Oral Med Oral Pathol. 1989;68:69-73.

14. Bertoni F, Unni KK, McLeod R, et al. Osteosarcoma resembling osteoblastoma. Cancer. 1985;55:416-26.

15. Bertoni F, Bacchini P, Donati D, et al. Osteoblastoma-like osteosarcoma the Rizzoli Institute experience. Mod Pathol. 1993;6: 707-16.

16. Abramovici L, Kenan S, Hytiroglou P, et al. Osteoblastoma-like osteosarcoma of the distal tibia. Skeletal Radiol. 2002;31:179-82.

17. Giannico G, Holt G, Homlar K, et al. Osteoblastoma characterized by a three-way translocation: report of a case and review of the literature. Cancer Genet Cytogenet. 2009;195:168-71.

18. Tani T, Okada K, Shoji K, et al. Osteoblastoma-like osteosarcoma. Skeletal Radiol. 2000;29:656-9.

19. Baker AC, Rezeanu L, Klein M, et al. Aggressive osteoblastoma: a case report involving a unique chromosomal aberration. Int $\mathrm{J}$ Surg Pathol. 2010;18(3):219-24. 